\title{
Influence of asymmetry and fissility on even-odd effect in fission-fragment yields
}

\author{
M. Caamaño ${ }^{1, a}$, F. Rejmund ${ }^{1, b}$, and K.-H. Schmidt ${ }^{1,2}$ \\ 1 GANIL, CNRS/IN2P3, CEA/DSM, Bd. Henri Becquerel, 14076 Caen, France \\ 2 GSI, Planckstrasse 1, 64291 Darmstadt, Germany
}

\begin{abstract}
Based on a wide systematics of fragment distributions measured in thermalneutron induced fission, the even-odd staggering in the fission-fragment element yields is investigated. The asymmetry evolution of the element yield distribution with the fissility of the fissioning nucleus is shown to be for an important part responsible for the decrease of the even-odd staggering with the fissility. The even-odd staggering close to symmetry is shown to be a small contribution to the global even-odd effect, and seems to vary little with the fissility of the nucleus. These experimental observations show that the established interpretation in which the intrinsic excitation energy at scission is accountable for the even-odd staggering amplitude has to be reconsidered.
\end{abstract}

\section{Introduction}

Even-odd staggering is a well-known characteristic of low-energy fission-fragment yields. The large amplitude of this staggering, which may reach $40 \%$ in the case of thorium [1], has always been fascinating to nuclear physicists. The observation of odd- $Z$ fragments from an even- $Z$ fissioning nucleus testifies the re-organisation of the intrinsic structure of the fissioning nucleus on its path towards scission. Indeed, in thermal-neutron induced fission of even- $Z$ actinides, the compound nucleus reaches the saddle deformation with an intrinsic excitation energy below the pairing gap. Thus, an ensemble of fully paired protons undergoes the deformation down to scission, where at this stage at least one pair is broken and both unpaired protons end up in different fission fragments to produce odd- $\mathrm{Z}$ species. The amplitude of the even-odd staggering is therefore linked to pair breaking and dissipation during the deformation in the fission process. It has always been a challenge to understand the mechanism of dissipation and the relation between pairing effects and the dissipated energy. The present work focuses on data for which nuclear charge yields are accessible. They emerge from experiments performed at ILL Grenoble for fissile actinides [2]. For the first time the even-odd structure in fragment yields is investigated over the complete systematic.

\section{Established interpretation of the even-odd effect}

The even-odd effect in low-energy fission fragment yields is usually understood as an evidence for pair breaking and is connected to the excitation energy gained during the nucleus deformation. It is assumed that if one pair at least is broken the even-odd staggering is null, as the protons of the broken

\footnotetext{
a Present address: Universidade de Santiago de Compostela, E-15706 Santiago de Compostela, Spain.

b e-mail: frejmund@ganil.fr
}

This is an Open Access article distributed under the terms of the Creative Commons Attribution-Noncommercial License 3.0, which permits unrestricted use, distribution, and reproduction in any noncommercial medium, provided the original work is properly cited. 

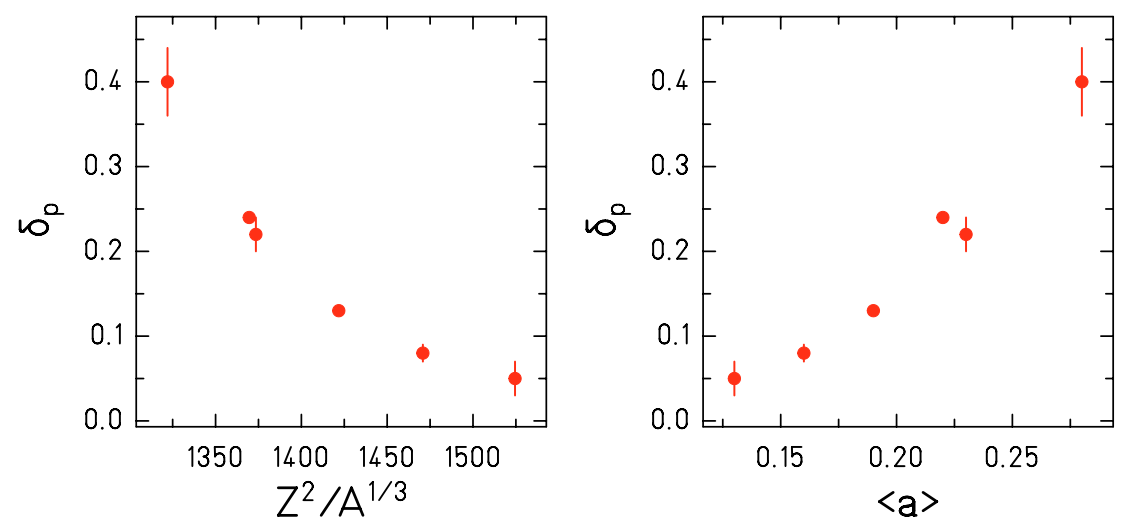

Fig. 1. Left panels: Global even-odd effect as a function of the Coulomb parameter, for the series of fragment distributions measured at ILL Grenoble (see text for details and references). Right panel: global even-odd effect as a function of the average asymmetry of the fragment distribution.

pair have equal probability to end-up each in one different fragment or in both fragments. Therefore, the amplitude of the even-odd effect in element yields is understood as being directly linked to the probability that all protons remain in a paired configuration at scission.

A simple method to quantify the pairing effect in the fragment production is to define the global even-odd staggering $\delta$ as the average difference between even- and odd- $Z$ yields over the full available distribution [1]. In the measurement of fragment yields of fissile actinides, it has been observed that the global even-odd staggering decreases with the fissility of the fissioning system [4]. The amplitude of the even-odd effect being associated to the dissipated energy gained by the nucleus, the decrease of the even-odd staggering with fissility has brought up the idea that more energy is dissipated in the descent from saddle to scission as the fissility of the fissioning nucleus increases. The fissility parameter $x=Z_{f}^{2} / A_{f}$ reflects the stability against fission, but its connection with the dissipated energy is not clear. On the other hand, the total energy release from saddle to scission may be expressed with a linear correlation with the Coulomb parameter $Z_{c}=Z_{f}^{2} / A_{f}^{1 / 3}[1]$ and therefore, the evolution of the even-odd staggering with the fissioning nucleus is usually investigated as a function of the Coulomb parameter $[5,6]$. The evolution of the global even-odd staggering is displayed in Fig. 1, left panel, as a function Coulomb parameters; a clear decrease is observed as the Coulomb parameter increases. The model proposed by Nifenecker et al. [7] correlates $\delta$ and the dissipated energy $E_{\text {diss }}$ with a very simple expression. This model estimates a dissipated energy of about $4 \mathrm{MeV}$ for ${ }^{230} \mathrm{Th}$ to $12 \mathrm{MeV}$ for ${ }^{250} \mathrm{Cf}$.

The local even-odd staggering $\delta(Z)$ is a measure of the deviation of the element distribution from a smooth behaviour, and is usually studied following the prescription of Tracy et al. [8]. For different fissioning systems [1,9-11], the even-odd staggering has been shown to be larger for large asymmetry. This experimental observation, reproduced in Fig. 2, has led to the notion of cold asymmetric fission, for which extreme deformation would take most of the available excitation energy, and consequently the intrinsic excitation energy would remain low [9]. However, no elaborate model exists to quantitatively describe the even-odd effect based on these assumptions.

\section{Limitation of the prevalent interpretation}

The general understanding of the even-odd effect briefly depicted above has been perturbed by the discovery in the late 90's of a large even-odd effect in the element distributions of odd-Z fissioning nuclei [12]. With experimental techniques based on inverse kinematics, the even-odd staggering has been measured for a large systematics of actinium and protactinium isotopes, over the complete $\mathrm{Z}$ distribution. In these odd- $Z$ systems, the probability to have at least one unpaired proton is always one. Assuming that any unpaired proton ends up in one or the other fragment with equal probability, 


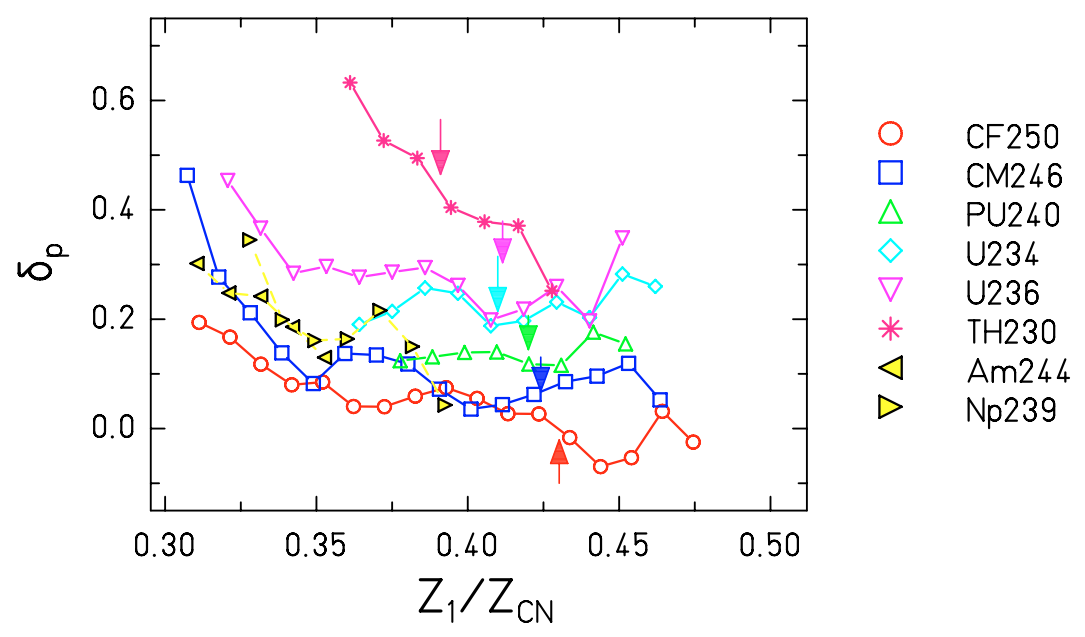

Fig. 2. Local even-odd effect for the series of element distributions of fissile nuclei.

it was expected that the even-odd effect would be zero over the complete element distribution. Its value was found to be zero close to symmetry and systematically increasing for large asymmetry, up to amplitudes as large as $40 \%$. In the heavier part of the fragment distribution, the even-odd staggering was found to be negative, revealing a higher probability for the unpaired nucleon to end up in the heavy fragment. The same observation of large even-odd effect has been reported in thermal-neutron induced fission of ${ }^{237} \mathrm{~Np}$ [13], and ${ }^{242 m} \mathrm{Am}$ [14]. The observation of an even-odd staggering for odd-Z fissioning nuclei reveals that the relation between the amplitude of the even-odd staggering in fission-fragment charge yields and the intrinsic excitation energy at scission is not as direct as depicted in the standard understanding as discussed above.

In addition to this experimental observation, which shows the limitation of the established interpretation, we consider in the present work the evolution of the fission fragment distribution with fissility. In low-energy fission of most actinides, shell effects induce an asymmetry in the mass and charge distributions of fragments, which show two groups [15]. The group of heavy fragments is distributed over an average value of $\mathrm{A} \sim 140$, independently of the mass of the fissioning system. In order to keep the total mass conservation, for heavier fissioning nucleus, the group of the light fragments moves towards heavier masses, approaching gradually the symmetry. The equivalent behaviour is observed in charge distributions. To explore the fission asymmetry, it is convenient to define the asymmetry parameter: $a=\left(Z_{H}-Z_{L}\right) / Z_{f}$, where $Z_{H}$ and $Z_{L}$ are the charge of heavy and light fragment, respectively. Precise information has been obtained for the light part of the asymmetric fission-fragment distribution [1,9-11,16-21]. They coincide with a heavy charge distribution centred on a constant value $Z=54$, independently of the fissioning nucleus, as was already reported in [22]. The distribution of the light fragments is gradually approaching the symmetry, as the fissioning system is getting heavier. Indeed, for the fissile systems investigated, the average asymmetry $\langle a\rangle$ of the light charge of the fission fragment distribution shifts from 0.28 to 0.13 when considering fissioning systems from ${ }^{230} \mathrm{Th}$ to ${ }^{250} \mathrm{Cf}$. In figure 1, right panel, the global even-odd staggering is displayed as a function of the average asymmetry of the light fragment distribution. A clear relationship is observed; when the average asymmetry decreases, the global even-odd effect decreases. The anti-correlation of the asymmetry of the fragment distribution with the mass (or the Coulomb parameter) of the fissioning system, associated to the clear influence of the asymmetry on the even-odd effect complicates the figure that was acknowledged up to now; it suggests that the decrease of the even-odd effect is not uniquely due to an increase of the excitation energy at the scission point.

Figure 2 shows an overview of the local even-odd effect for all systems investigated at ILL in thermal neutron induced fission. This figure shows the general trend of an increase of the even-odd effect with larger asymmetry, as discussed above. The rate of increase is largest for the lightest system ${ }^{230} \mathrm{Th}$ and decreases for the heavier systems. The even-odd effect for the different systems approach each 
other at symmetry, but due to missing experimental information at symmetry and due to the scattering of the data points one can only say that they converge to a rather narrow range between 0 and 20 percent. For several systems, the data points that are closest to symmetry present appreciably higher local even-odd effect than expected from the global trend. This effect may be associated to the influence of the $\mathrm{Z}=50$ shell in the complementary fragment, which is known to enhance the yield of tin isotopes and, thus, leads to a local increase of the deduced even-odd effect, because its derivation cannot distinguish between structures caused by pairing or shell effects. The even-odd effect of the odd $\mathrm{Z}$ fissioning systems at large asymmetry follows the general behaviour of the even $\mathrm{Z}$ fissioning systems. Their values are very close to those of the neighbouring even $\mathrm{Z}$ systems, as was reported already in [13]. As discussed in the previous section, the even-odd effect in odd $\mathrm{Z}$ fissioning system can only be understood by a higher probability for the unpaired proton to end up in the heavy fragment. Since there is no preference for unpaired protons to end up in one or the other fragment in symmetric fission by trivial reasons, the even-odd effect in odd $\mathrm{Z}$ fissioning system is necessarily zero for symmetric splits. In these odd $\mathrm{Z}$ fissioning systems, an even odd effect may only be generated by a mechanism which is related to some difference in the properties of the fragments in asymmetric splits. Due to the similarity of the values of even $\mathrm{Z}$ and odd $\mathrm{Z}$ systems one may conclude that the same mechanism is responsible for the increase of the even-odd effect in even $\mathrm{Z}$ fissioning systems. These experimental observations lead to the conclusion that the conventionally assumed relation between pair breaking and even-odd effect in the fission yields is valid only close to symmetry, and that it is limited to a restricted value below $20 \%$, which does not seem to varying much as the fissility (or Coulomb parameter) of the fissioning system varies. Most of the amplitude of the even-odd effect is caused by the asymmetry component of the fragment distribution, and is common to the odd- $\mathrm{Z}$ and even- $\mathrm{Z}$ fissioning systems.

\section{Conclusion}

A systematic investigation on even-odd staggering in fission-fragment yields based on thermal-neutroninduced fission of fissile nuclei is reported. It is shown that the global even-odd effect cannot be considered to derive conclusions on dissipation, as it includes a strong contribution of the asymmetry of the scission split, which has to be taken into account to explain the amplitude of the global even-odd effect. As a consequence, the previous models [7,23], which aimed at describing the even-odd structure without considering the influence of the fission-fragment phase space, are not suitable to derive realistic conclusion on the dissipated energy. Finally, the local even-odd effect at asymmetry measured in thermal-neutron induced fission for even- or odd-Z fissioning systems present the similar trend to decrease with increasing fissility or Coulomb parameter of the fissioning nucleus. The local even-odd effect at asymmetry is varying gradually with the Coulomb parameter, showing that the same process is responsible for the even-odd effect in asymmetric splits, independently of the evenness or oddness of the fissioning nucleus. For even-Z fissioning systems, the local even-odd effect close to symmetry is shown to be essentially independent of the fissioning system, which forces to revisit the previously established influence of the fissioning system on pair-breaking.

\section{References}

1. J.-P. Bocquet, R. Brissot, H.R. Faust, M. Fowler, J. Wilhelmy, M. Asghar, M. Djebara, Zeit. für Phys. A 335 (1990) 41

2. F. Rejmund, M. Caamaño, K.-H. Schmidt, GANIL Report 10-01, http://hal.in2p3.fr/in2p300487186/fr/, and references therein

3. K.-H. Schmidt et al., Nucl. Phys. A 665 (2000) 221

4. F. Gönnenwein, “The Nuclear Fission Process", Ed. C. Wagemans, CRC Press, 1991

5. F. Rejmund, A.V. Ignatyuk, A.R. Junghans, K.-H. Schmidt, Nucl. Phys. A 678 (2000) 215

6. H. Naik, S.E Dange, R.J. Singh, S.B. Manohar, Nucl. Phys. A 612 (1997) 143

7. H. Nifenecker, G. Mariolopoulos, J. P. Bocquet, R. Brissot, Mme Ch. Hamelin, J. Crancon, Ch. Ristori, Zeit. für Phys. A 308 (1982) 39 
8. B.L. Tracy, J. Chaumont, R. Klapisch, J.M. Nitschke, A.M. Poskanzer, E. Roeckl, C. Thibault, Phys. Rev. C 5 (1972) 222

9. J.L. Sida , P. Armbruster, M. Bernas, J.P. Bocquet, R. Brissot, H.R. Faust, Nucl. Phys. A 502 (1989) 233c

10. R. Hentzschel, H.R. Faust, H.O. Denschlag, B.D. Wilkins, J. Gindier, Nucl. Phys. A 571 (1994) 427

11. D. Rochman, I. Tsekhanovich, F. Gönnenwein, V. Sokolov, F. Storrer, G. Simpson, O. Serot, Nucl. Phys. A 735 (2004) 3

12. S. Steinhäuser et al., Nucl. Phys. A 634 (1998) 89

13. I. Tsekhanovich, H.-O. Denschlag, M. Davi, Z. Büyükmumcu, F. Gönnenwein, S. Oberstedt, H.R. Faust, Nucl. Phys. A 688 (2001) 633

14. I. Tsekhanovich, H.-O. Denschlag, M. Davi, Z. Büyükmumcu, M. Woestheinrich, F. Gönnenwein, S. Oberstedt and H.R. Faust, Nucl. Phys. A 658 (1999) 217

15. J.P. Unik, J.E. Gindler, L.E. Glendenin, K.F. Flynn, A. Gorski, R.K. Sjoblom, Proc. Symp. Phys. Chem. Fission, Rochester 1973, IAEA Vienna (1974), vol. 2, p. 19

16. M. Djebara, M. Asghar, J.P. Bocquet, R. Brissot, M. Maurel, H. Nifenecker, Ch. Ristori, Nucl. Phys. A 425 (1984) 120

17. D. Rochman, H. Faust, I. Tsekhanovich, F. Gönnenwein, F. Storrer, S. Oberstedt, V. Sokolov, Nucl. Phys. A 710 (2002) 3

18. W. Lang, H.-G. Clerc, H. Wolfarth, H. Schrader, K.-H. Schmidt, Nucl. Phys. A 345 (1980) 34

19. U. Quade et al., Nucl. Phys. A 487 (1988) 1

20. C. Schmitt et al., Nucl. Phys. A 430 (1984) 21

21. M. Djebara et al, Nucl. Phys. A 496 (1989) 346

22. C. Böckstiegel, S. Steinhäuser, K.-H. Schmidt, H.-G. Clerc, A. Grewe, A. Heinz, M. de Jong, A.R. Junghans, J. Müller, B. Voss, Nucl. Phys. A 802 (2008) 12

23. B. Bouzid, M. Asghar, M. Djebara, M. Medkour, J. Phys. G: Nucl. Part. Phys. 24 (1998) 1029 